\title{
EXPONENTIAL ESTIMATES IN AVERAGING AND HOMOGENISATION
}

\author{
KARSTEN MATTHIES
}

\begin{abstract}
Many partial differential equations with rapid spatial or temporal scales have effective descriptions which can be derived by homogenisation or averaging. In this article we deal with examples, where quantitative estimates of the error is possible for higher order homogenisation and averaging. In particular, we provide theorems, which allow homogenisation and averaging beyond all orders by giving exponential estimates of appropriately averaged and homogenised descriptions. Methods include iterated averaging transformations, optimal truncation of asymptotic expansions and highly regular solutions (Gevrey regularity). Prototypical examples are reaction-diffusion equations with heterogeneous reaction terms or rapid external forcing, nonlinear Schrödinger equations describing dispersion management, and second-order linear elliptic equations.
\end{abstract}

\section{INTRODUCTION}

Many classical multiscale problems are modelled by (partial) differential equations which are heterogeneous in space (explicitly depending on the space variable $x$ ) or nonautonomous (explicitly dependent on time $t$ ). The multiscale character is introduced, if these dependencies are rapid, i.e. they are on a fast scale. Typically, this is achieved by introducing a small parameter $\varepsilon$ and considering dependencies of the form $x / \varepsilon$ or $t / \varepsilon$. Application areas, where such descriptions are used, include continuum mechanics, chemical reactions, nonlinear optics, ecology, and celestial mechanics among others. The $x / \varepsilon$ describes e.g. the underlying varying microstructure of the medium, whereas an $t / \varepsilon$ dependency is used for rapid external excitations.

Then there are two main approaches to such problems when comparing the heterogeneous/ nonautonomous partial differential equations with their homogeneous/ autonomous counterparts. Firstly one can identify effects that are created by the multiscale structure. Secondly one can try to find effective descriptions by homogeneous and autonomous equations without any explicit multiscale structure. This process is usually called homogenisation for space-dependent problems and averaging for time-dependent problems. Of course, these two approaches are related, as effective descriptions can be used to estimate the size of effects.

There are many different methods to derive the homogenised or averaged descriptions, these can be grouped roughly into two groups. The first one is using weak convergence methods (see e.g. [Tar79, Tar86, Bor98, GMMP97, JKO94]), which are applicable to many problems but which usually do not provide a quantitative bound on the effectiveness, i.e. on the error of approximation. The next group of methods is based on asymptotic expansions in the small parameter $\varepsilon$. When ruling out purely formal methods, then the rigorous asymptotic techniques can be also used to derive 
quantitative error bounds on the approximation error. These will be then in some finite or even exponential order in $\varepsilon$. Some classical methods can be found in [AKN97, BLP78, BP84, JKO94, LM88]. To derive such estimates, the finer analysis often also requires additional assumptions on the structure of the differential equation.

Besides certain regularity assumptions on the solutions, we will mainly consider cases where the underlying microstructure and external excitation is either periodic or quasiperiodic. When expanding a rigorous finite order procedure into a series, even in a very benign example, like periodic averaging of analytic ordinary differential equations

$$
\dot{x}=f(x, t / \varepsilon),
$$

convergence of the expansion cannot be expected in general. Nevertheless, beyond a finite asymptotic expansion, there are exponential estimates in several aspects, early examples are by Nekhoroshev, and Neishtadt; see, e.g., [Nek79, Nei84, LM88]. These then yield upper estimates on all kinds of effects created by the periodic nonautonomous structure.

Here, the purpose of this article is to describe a number of asymptotic techniques which provide effective descriptions up to exponentially small errors for wide classes of multiscale problems. The remainder of the article has the following structure. First we will provide a list of prototypical partial differential equations and interesting solutions involving multiple scales in section 2 . Then we will describe several finite order methods like classical averaging, homogenisation and their relation to normal forms in the dynamical systems literature (section 3). Detailed results on techniques for upper exponential estimates for the earlier examples will be given in section 4 . In section 5 , we will use these results to estimate several possible effects. We conclude with a discussion, where we are e.g. describing some situations, where lower estimates are possible, section 6 .

\section{EXAMPLES}

We provide some typical examples for which effective descriptions beyond every order will be possible. For larger classes of examples and more general assumptions, we refer to the relevant papers [Mat01, MS03, Mat04, KMS06].

2.1. Partial differential equations with rapid time dependence. The basic example of nonautonomous differential equations is the periodic ordinary differential equation

$$
\dot{u}=f(u, t / \varepsilon) \text { with } f(., \tau)=f(., \tau+1) \text { for all } \tau \in \mathbf{R}
$$

To describe more general dependencies than the purely periodic one, we introduce the phase $\phi \in$ $T^{m}=(\mathbf{R} / \mathbf{Z})^{m}, m \in \mathbf{N}$. Then we rewrite the autonomous equation as

$$
\begin{aligned}
\dot{u} & =f(u, \phi) \\
\dot{\phi} & =\frac{1}{\varepsilon} \Omega(u, \phi)
\end{aligned}
$$


with $\Omega(u, \phi) \geq c_{0}>0$. Then $m=1, \Omega(u, \phi)=1$ recovers the periodic case. Results on averaging in this situation, under certain assumptions on $\Omega$, can be found in [BoMi61, SV85]. Exponential estimates are due to Neishtadt [Nei84] for $m=1$ and to Simó [Sim94] for $m>1$ and

$$
\begin{aligned}
& \Omega(u, \phi)=\omega \in \mathbf{R}^{m} \text { with Diophantine conditions on } \omega \\
&|(\ell, \omega)| \geq \gamma|\ell|^{-\tau} \text { for some } \gamma>0, \tau>m-1 \text { and all } \ell \in \mathbf{Z}^{m}
\end{aligned}
$$

Partial differential equations with such a structure can be found in the context of systems of reaction-diffusion equations

$$
\begin{aligned}
u_{t} & =D \Delta u+f(u)+g(u, \phi) \\
\dot{\phi} & =\frac{1}{\varepsilon} \Omega(u, \phi) \\
(u(0), \phi(0)) & =\left(u_{0}, \phi_{0}\right) \in X \times T^{m}
\end{aligned}
$$

with $D=\operatorname{diag}\left(d_{1}, \ldots, d_{n}\right)$. We will consider the functiion $u$ on $[0,1]^{d}$ with periodic boundary conditions. Initial conditions are in the phase space $X=H_{p e r}^{s}\left([0,1]^{d}, \mathbf{R}^{n}\right)$ with $s>d / 2$ to ensure the embedding of $X$ into $C^{0}$ and differentiability of the nonlinearities in $X$. Reaction diffusion equations are the prime example for pattern formation, for a review see [FS03]. An external forcing can be introduced in light sensitive reactors by periodic changes, see e.g. [SaScWu99, RMMSC03] for a framework and an example.

Another example are nonlinear Schrödinger type equations, which e.g. describe the evolution of pulses in optical fibres [NM92]. The evolution then describes the changes of the pulse while propagating along the fibre, so changes in the material due to dispersion management and localised amplification are described as

$$
\begin{aligned}
\mathrm{i} u_{t} & =d(\phi) u_{x x}+C(\phi,|u|) u \\
\dot{\phi} & =\frac{1}{\varepsilon} \Omega(u)+g(u, \phi)
\end{aligned}
$$

As a phase-space we use as in (2.4) some Sobolev space, denoted again by $X$. Variants describing general interaction between fast oscillations and pulses can be also described in the setting of Hamiltonian PDE. For the necessary frame work and the description of non-adiabatic coupling, we refer the reader to [MS03].

2.2. Partial differential equations with rapid space dependence. First we consider a heterogeneous version of (2.4), where the heterogeneity is in the reaction term, examples of these can be found in several modelling areas [BHR05, Kee00, KS98]:

$$
u_{t}=\Delta_{x, y} u+f(u, x / \varepsilon)
$$

We will be in particular interested in the behaviour in infinite cylinders, i.e. $x \in \mathbf{R}$ and $y \in \Sigma$ with $\Sigma$ a bounded cross-section. In particular, let $\Sigma=[0,1]^{d}$ with periodic boundary conditions in $y$. 
When looking for stationary solutions, we obtain

$$
\Delta_{x, y} u+f(u, x / \varepsilon)=0 .
$$

We rewrite the equation and use the idea of spatial dynamics. It is a way to construct special solutions to PDE on unbounded domains. For this we let

$$
U=\left(\begin{array}{c}
u \\
u_{x}
\end{array}\right) ; \quad A=\left(\begin{array}{cc}
0 & I \\
-\Delta_{y} & 0
\end{array}\right) ; \quad F(U, x / \varepsilon)=\left(\begin{array}{c}
0 \\
-f(u, x / \varepsilon)
\end{array}\right)
$$

Renaming $x$ as time $t$, we again obtain an equation

$$
U_{t}=A U+F(U, t / \varepsilon),
$$

which has the form of a rapidly forced evolution equation. The phase space $X$ is a function space on the cross-section $\Sigma$ like $X=H^{s+1}\left(\Sigma, \mathbf{R}^{n}\right) \times H^{s}\left(\Sigma, \mathbf{R}^{n}\right)$. Even if the Cauchy problem is not well-posed, this method of spatial dynamics has a long history, see [Kir82] and further work (see, e.g.[IM91, AM95, FS03] and the references therein).

When considering travelling waves in heterogeneous media one is using the ansatz

$$
u(x, y, t)=v(x-c t, y, x / \varepsilon),
$$

i.e. the profile $v$ of the travelling wave is changing periodicly while moving through the periodic medium. This can be also formulated as a spatial dynamics problem, for details see [MSU06].

Variants also include heterogeneities in the main part like in classical homogenisation theory. The homogenisation of heterogeneous second-order elliptic equations is appearing in many stationary problems, consider

$$
-\nabla \cdot(\mathcal{A}(x / \varepsilon) \nabla u)(x))=f(x) .
$$

The matrix $\mathcal{A} \in L^{\infty}\left(T^{d}\right)$ on $T^{d}=(\mathbf{R} / \mathbf{Z})^{d}$ is assumed to be symmetric and uniformly elliptic. Furthermore we assume boundary conditions for $x$ in some bounded domain, here again periodic boundary conditions.

\section{Finite ORDER ESTIMATES AND NORMAL FORMS}

The basic idea of this approach is to transform the equation to derive an effective, simpler version of the differential equation. The method proved to be very successful in the analysis of finite dimensional dynamical systems [LM88, SV85]. Now we consider problems, that can be written as an evolution equation with external forcing like $(2.4,2.8)$ and under further assumption also (2.5) (see [Mat04]). More examples and references can be found in [Mat04, Ver05, Ver06], including other variants of explicitly time dependent partial differential equations and other near-identity transformations to obtain the form in (3.10). We consider

$$
\begin{aligned}
u_{t} & =A u+f(u, \phi) \\
\dot{\phi} & =\frac{1}{\varepsilon} \Omega(u, \phi),
\end{aligned}
$$


where $u \in X$ for some appropriate phase space $X$. Then a near-identity transformation on a ball $B_{R}(X)$ can be written in the form

$$
u=v+\varepsilon W(v, \phi)
$$

The transformed equation can be derived from

$$
\partial_{t} u=\partial_{t} v+\varepsilon \partial_{v} W(v, \phi) \partial_{t} v+\varepsilon \partial_{\phi} W(v, \phi) \partial_{t} \phi
$$

then

$$
\begin{aligned}
\partial_{t} v=(I & \left.+\varepsilon \partial_{v} W(v, \phi)\right)^{-1}\{A(v+\varepsilon W(v, \phi))+f(v+\varepsilon W(v, \phi), \phi) \\
& \left.-\varepsilon \partial_{\phi} W(v, \phi) \frac{1}{\varepsilon} \Omega(v, \phi)\right\} .
\end{aligned}
$$

So depending on the form of $\Omega$, one can try to reduce the externally forced term $f$ by an appropriate choice of $W$. It is notational convenient to split $f$ such that

$$
\begin{aligned}
f(v, \phi) & =\hat{f}(v)+g(v, \phi) \text { such that } \\
\langle g\rangle & =\int_{T^{m}} g(v, \psi) \mathrm{d} \psi=0 .
\end{aligned}
$$

In the simplest case of periodic external forcing, i.e. $m=1, \Omega \equiv 1$, we let

$$
W(v, \phi)=\int_{0}^{\phi} g(v, \psi) \mathrm{d} \psi
$$

then the transformed equation has the form

$$
\begin{aligned}
\partial_{t} v=(I+ & \left.\varepsilon \partial_{v} W(v, \phi)\right)^{-1}\{A(v+\varepsilon W(v, \phi))+\hat{f}(v+\varepsilon W(v, \phi)) \\
& +g(v+\varepsilon W(v, \phi), \phi)-g(v, \phi)\} .
\end{aligned}
$$

This removes the lowest order nonautonomous terms. A problem is to estimate the remainder $r$, if we rewrite the equation as

$$
v_{t}=A v+\bar{f}(v ; \varepsilon)+r(v, \phi ; \varepsilon) .
$$

The remainder involves terms depending on the unbounded operator $A$, such that $r$ is only formally small. A more promising variant is to use some bounded Galerkin type approximation to perform the estimates, where the Galerkin approximation $v_{N}$ is chosen depending on $\varepsilon$. For all our examples, there exists a sequence of (Galerkin) projections $\left(P_{N}\right)_{N \in \mathbf{N}}$ which satisfy

(i) the sequence of projections converges strongly to the identity on the phase space $X$,

$$
\lim _{N \rightarrow \infty} P_{N} u=u \text { in } X \text { for all } u \in X
$$

(ii) the projections $P_{N}$ commute with $A$ on its domain of definition

$$
P_{N} A u=A P_{N} u \text { for all } u \in D(A)
$$


(iii) the operator $A$ is bounded on $\operatorname{Rg} P_{N}$,

$$
\left|A P_{N} u\right|_{X} \leq N\left|P_{N} u\right|_{X} \text { for all } u \in X .
$$

The equation with projection $P_{N}$ on the approximation space is

$$
\partial_{t} u_{N}=A u_{N}+P_{N} f\left(u_{N}, \phi\right) .
$$

Then the transformed equation is

$$
\begin{gathered}
\partial_{t} v_{N}=\left(I+\varepsilon \partial_{v_{N}} W\left(v_{N}, \phi\right)\right)^{-1}\left\{A\left(v_{N}+\varepsilon W\left(v_{N}, \phi\right)\right)+f\left(v_{N}+\varepsilon W\left(v_{N}, \phi\right), \phi\right)\right. \\
\left.-\varepsilon \partial_{\phi} W\left(v_{N}, \phi\right) \frac{1}{\varepsilon} \Omega\left(v_{N}, \phi\right)\right\} .
\end{gathered}
$$

Regrouping again yields

$$
\partial_{t} v_{N}=A v_{N}+\bar{f}_{N}\left(v_{N} ; \varepsilon\right)+r_{1}\left(v_{N}, \phi ; \varepsilon\right)
$$

then the remainder term is of order

$$
\sup _{\left\|v_{N}\right\| \leq R} \varepsilon\left\|A v_{N}\right\|\left\|g\left(v_{N}, \phi\right)\right\|,
$$

for $V$ in a large ball $B_{R}(X)$. The remainder is small for an appropriate choice of $N(\varepsilon)$. E.g. when we are choosing $N(\varepsilon)$ such that $\left\|A v_{N}\right\| \sim \varepsilon^{-1 / 2}$, we obtain a rigorous estimate on $r_{1} \in \mathcal{O}\left(\varepsilon^{1 / 2}\right)$ in the approximation space $P_{N} X$, this is uniform for $\varepsilon \rightarrow 0, N(\varepsilon) \rightarrow \infty$.

The important property is now, that (3.18) has still the form of the original equation (3.16). So the equation can be transformed again to obtain a new remainder term $r_{2}$. This is of order $\sup _{\left\|v_{N}\right\| \leq R} \varepsilon\left\|A v_{n}\right\|\left\|r_{1}\left(v_{N}, \phi\right)\right\|$, such that $r_{2} \in \mathcal{O}(\varepsilon)$ for the same choice of $N(\varepsilon)$ with $\varepsilon \rightarrow 0, N(\varepsilon) \rightarrow$ $\infty$. The transformed equation has again the same form. Hence this procedure can be iterated to obtain arbitrary finite order estimate $\mathcal{O}\left(\varepsilon^{k}\right)$, provided we can ensure enough regularity of the nonlinearity.

In a detailed analysis taking into account all parts of the remainder term one can see, that the constant in the $\mathcal{O}\left(\varepsilon^{k}\right)$ remainder will become large with $k$. So this procedure will not lead to a convergent asymptotic expansion. But keeping track of the constants in the remainder estimates depending on $k$ and $\varepsilon$ is crucial for later exponential estimates.

Here of course, the error of the Galerkin approximation is still to be estimated. When extending the transformation back to the full space by

$$
u=v+\varepsilon P_{N} W\left(P_{N} v, \phi\right)
$$

this leads to

$$
\partial_{t} v=A v+\bar{f}(v ; \varepsilon)+r(v, \phi ; \varepsilon)
$$

with additional terms in $\bar{f}$ and $r$ due to the Galerkin approximation. The additional terms are all of the form $G\left(v-P_{N} v\right)$. 
Another situation with an explicit choice of $W$ is for $m=1$ and $\Omega(v, \phi)=\bar{\Omega}(v)+\varepsilon \beta(v, \phi)$. The phase $\phi$ will also be transformed. We choose the explicit change of coordinate $\left(u_{N}, \phi\right)=$ $\left(v_{N}, \psi\right)+\varepsilon W\left(v_{N}, \psi\right)$

$$
W\left(v_{N}, \psi ; \varepsilon\right)=\left(\begin{array}{c}
W^{1}\left(v_{N}, \psi ; \varepsilon\right) \\
W^{2}\left(v_{N}, \psi ; \varepsilon\right)
\end{array}\right),
$$

with

$$
W^{1}\left(v_{N}, \psi ; \varepsilon\right)=\frac{1}{\bar{\Omega}\left(v_{N}\right)} \int_{0}^{\phi} g\left(v_{N}, \tau\right) \mathrm{d} \tau
$$

and

$$
\begin{aligned}
W^{2}\left(v_{N}, \psi ; \varepsilon\right)= & \frac{1}{\bar{\Omega}\left(v_{N}\right)} \int_{0}^{\phi} \beta\left(v_{N}, \tau\right) \\
& +\partial_{v_{N}} \bar{\Omega}\left(v_{N}\right)\left(W_{k+1}^{1}\left(v_{N}, \tau\right)-\left\langle W^{1}\left(v_{N}, .\right)\right\rangle\right) \mathrm{d} \tau
\end{aligned}
$$

where $\langle$.$\rangle again denotes the T^{1}$-average.

The third situation is the quasiperiodic case, i.e. $m>1$ and $\Omega(v, \phi)=\omega$ with $\omega=\left(\omega_{1}, \ldots, \omega_{m}\right)$ fulfilling Diophantine conditions: there are constants $\gamma>0$ and $\tau>m-1$ such that for all $\ell \in \mathbf{Z}^{p}$

$$
|(\ell, \omega)| \geq \gamma|\ell|^{-\tau}
$$

as for the finite dimensional result (2.3). Then the transformation is given terms of the Fourier expansion of the phase dependent term. Letting

$$
g\left(u_{N}, \phi ; \varepsilon\right)=\sum_{\ell \in \mathbf{Z}^{p}} g_{\ell}\left(u_{N} ; \varepsilon\right) \exp (\mathrm{i} 2 \pi(\ell, \phi))
$$

then we transform $u_{N}=v_{N}+\varepsilon W\left(v_{N}, \phi ; \varepsilon\right)$ with

$$
W\left(v_{N}, \phi ; \varepsilon\right)=\sum_{\ell \in \mathbf{Z}^{p}} \frac{g_{\ell}\left(v_{N} ; \varepsilon\right)}{2 \pi \mathrm{i}(\ell, \omega)} \exp (\mathrm{i} 2 \pi(\ell, \phi))
$$

Classical homogenisation theory of (2.9) gives an asymptotic expansion (cf. e.g. [BP84]):

$$
u^{\varepsilon, N}(x)=\sum_{n=0}^{N+2} \varepsilon^{m} u^{(n)}(x, x / \varepsilon),
$$

where the functions $u^{(l)}(x, y)$ are required to be periodic in the fast variable $y$. For this problem one can construct in this way a full asymptotic expansion with $u^{(l)}$ adopting the following form (see e.g. [BP84], [CS04]):

$$
u^{(n)}(x, y)=\sum_{l=0}^{n} \sum_{|k|=l} N_{k}(y) D_{x}^{k} v_{n-l}(x),
$$

where $N_{0}(y) \equiv 1$ and $N_{k}(y)$ are periodic solutions of the main $(|k|=1)$ and higher order $(|k|>1)$ unit cell problems in $y$. The functions $v_{s}(x), s \geq 0$, solve certain recurrent systems of equations in $x$, see [BP84, KMS06]. This cannot be easily rephrased as an iterative procedure but the idea of tracking the dependence of the constants and taking expansion depending on $\varepsilon$ can also be used in 
the context of (2.9). For other quantitative estimates on homogenisation involving quasiperiodic terms see [FV01].

The method of iterative transformation procedures are encountered widely in dynamical systems theory as the concept of normal forms, see e.g. [AKN97, Van89]. An important question is the description of the behaviour near an equilibrium of

$$
\dot{u}=A u+f(u), u \in \mathbf{R}^{n},
$$

where $f$ is of higher order in $u$. A particular simple form is sought for $f$. If $A$ has purely imaginary spectrum and is semisimple (i.e. there are no Jordan blocks of size 2 or bigger in its complex Jordan normal form), then we obtain a normal form transformation by averaging, see [Van89][Sec.2.4]. When we assume that $\exp (A t)$ is periodic with period $T$, then all terms can be removed by appropriate coordinate changes, except those which are invariant under the averaging operator

$$
\pi f(u)=\frac{1}{T} \int_{0}^{T} \exp (-A t) f(\exp (A t) u) \mathrm{d} t
$$

for all $u \in \mathbf{R}$. But while removing terms, which are not invariant under $\pi$, one also changes certain higher order invariant terms, such that pure averaging will not give a correct normal form.

The relation of averaging and purely imaginary spectrum has also been used in the context of wave equations and similar partial differential equations, see [Bam03a, Bam03b, Bam06, Kr89, Ver05, Ver06]. Here also the reductions to Galerkin approximations were used depending on a small parameter, which is introduced via scaling $u \mapsto \varepsilon u$ to obtain

$$
\partial_{t} u=A u+\varepsilon \tilde{f}(u, \varepsilon),
$$

with $\tilde{f}(u, \varepsilon)=1 / \varepsilon^{2} f(\varepsilon u) \in \mathcal{O}(1)$. The equation is then simplified by averaging the semigroup $\exp (A t)$. Here the name 'Galerkin averaging' was introduced. The development of 'Exponential averaging' in [Mat01, MS03] was independent of this.

\section{Exponential estimates}

We will now collect methods to move from finite order normal form and averaging transformations to exponential estimates in the framework described above. In particular, we obtain results to all orders $\mathcal{O}\left(\varepsilon^{k}\right)$ and beyond. In the last section, we derived iterative estimates on the Galerkin approximation space $P_{N} X$, and obtained error terms due to the Galerkin approximation. We will control both errors at the same time. There are three variables to choose to minimise the error. Firstly there is $\varepsilon$, which is given, then we have the choice of the number of normal form steps and there is also the index $N$ of the Galerkin approximation.

The crucial ingredient, which dictates the optimal coupling, is the dependence of the error on $N$, which is in our examples a question of regularity. As it is a question about the decay of spatial Fourier coefficients for periodic boundary conditions or the decay of the Fourier transform for problems on $\mathbf{R}^{d}$. A class of function spaces is introduced to capture this. We define the 
Gevrey space in the following way: Assuming that there exists a closed, densely defined, boundedly invertible operator $\Gamma_{\sigma, p}$ with domain of definition

$$
\mathcal{G}_{\sigma, \nu}:=D\left(\Gamma_{\sigma, \nu}\right) \subset D(A)
$$

such that $\operatorname{Rg} P_{N} \subset \mathcal{G}_{\sigma, \nu}, \Gamma_{\sigma, \nu}\left(\operatorname{Rg} P_{N}\right)=\operatorname{Rg} P_{N}$ for all $N$, and

$$
\Gamma_{\sigma, \nu} A u=A \Gamma_{\sigma, \nu} u \text { for all } u \in \operatorname{Rg} P_{N} .
$$

We equip the Gevrey spaces $\mathcal{G}_{\sigma, \nu}$ with the graph norm

$$
|u|_{\mathcal{G}_{\sigma, p}}=|u|_{X}+\left|\Gamma_{\sigma, \nu} u\right|_{X}
$$

For the theorem, we will assume that Gevrey-regular functions $u \in G_{\sigma, \nu}$ are exponentially well approximated by the Galerkin projections $P_{N}$, i.e.

$$
\left|\Gamma_{\sigma, \nu}^{-1}\left(I-P_{N}\right)\right| \leq C_{0} \exp \left(-\sigma / N^{\nu}\right),
$$

for $N$-independent constants $C_{0}(\sigma, \nu)$. Now we are in a position to formulate the following theorems about exponential estimates.

Theorem 1 (Exponential averaging of parabolic equation under periodic forcing [Mat01]). Let the nonlineairties $f, g$ be entire functions on $\mathbf{R}^{n}$, let $g$ be continuous on $T^{1}$ and let $\Omega \equiv 1$ in equation (2.4). Then the equation can be transformed on bounded sets in $X$ by a real analytic and time-periodic change of coordinates for $0<\varepsilon<\varepsilon_{0}$

$$
u=v+\varepsilon W(v, t / \varepsilon ; \varepsilon)
$$

with $W$ bounded on any ball of radius $R$ in $X$. The transformed nonautonomous terms $r$ are exponentially small after a short transient, but the equation may contain nonlocal terms $\bar{f}$ :

$$
\frac{\partial}{\partial t} v(x, t)=D \Delta v(x, t)+f(v(x, t))+\bar{f}(v(t) ; \varepsilon)(x)+r(v(t), t / \varepsilon ; \varepsilon)(x),
$$

with $v(0)=u_{0},|v(0)|_{X}<R$ and $t \in\left(0, t^{*}\right)$

$$
\begin{aligned}
& \sup _{|v(0)|_{X}<R}|\alpha(v(t))|_{X} \leq C \varepsilon \exp \left(-\min (t, c) \varepsilon^{-1 / 3}\right) \\
& \sup _{|v(0)|_{X}<R}|\bar{f}(V(t))|_{X} \leq C \varepsilon+C \exp \left(-\min (t, c) \varepsilon^{-1 / 3}\right)
\end{aligned}
$$

where $C, c, \varepsilon_{0}$ do not depend on $u_{0}$.

Proof. For a detailed proof see [Mat01]. We will sketch the proof using the transformation of (3.16) in the previous section. Adapting the results of [Nei84] and a coupling

$$
N(\varepsilon) \varepsilon^{\alpha}=1
$$

we can estimate the remainder term in the approximation space $P_{N} X$. For this we use a complex extension and a Cauchy estimate. Performing $k=\left[\varepsilon^{-1+\alpha}\right]$ transformation steps as in (3.18) and 
proving that $\left|r_{j+1}\right| \leq\left|r_{j}\right| / 2$ for $j=1, \ldots, k$ yields that the remainder term on the approximation space is

$$
\left|r_{k}\right| \leq C 2^{-k} \leq C \exp \left(-c \varepsilon^{-1+\alpha}\right)
$$

for an appropriate choice of $C$ and $c$. The autonomous correction term is at most of order $\mathcal{O}(\varepsilon)$. To estimate the effect of the transformation on the full space $X$, we use a regularity result for equation (2.4). Letting $A=\Delta$ and

$$
\Gamma_{\sigma, \nu}=\exp \left(-\sigma|A|^{\nu}\right)
$$

we obtain that

$$
u(t) \in \begin{cases}\mathcal{G}_{t, 1 / 2} & \text { for } t \in\left[0, t^{*}\right] \\ \mathcal{G}_{t^{*}, 1 / 2} & \text { for } t>t^{*}\end{cases}
$$

as long as $|u(t)|_{X}$ remains bounded. This and similar results can be found in [Pro91, TBDHT96, FT98], adaptions to equations with nonlocal operators as they appear in the transformed equations can be found in [Mat01]. Using this regularity result and (4.26), we can estimate the additional error terms due the Galerkin approximation of the form $G\left(v-P_{N} v\right)$. Thus the overall remainder can be bounded by

$$
\begin{aligned}
& \left|r_{k(\varepsilon)}\right|_{X}+\left|G\left(v(t)-P_{N(\varepsilon)} v(t)\right)\right|_{X} \\
\leq & C \exp \left(-c \varepsilon^{-1+\alpha}\right)+C_{0} \exp \left(-\min \left(t, t^{*}\right) / N(\varepsilon)^{1 / 2}\right) \\
= & C \exp \left(-c \varepsilon^{-1+\alpha}\right)+C_{0} \exp \left(-\min \left(t, t^{*}\right) \varepsilon^{-\alpha / 2}\right) .
\end{aligned}
$$

Choosing $\alpha=2 / 3$ yields the desired result for an appropriate choice of $C$ and $c$. The other results including the estimate on $\bar{f}$ are direct consequences of the detailed analysis in [Mat01].

So far the analysis was about the equation, now we compare the solutions of (4.28) with solutions of the truncated equation,

$$
\partial_{t} u=\Delta u+f(u)+\bar{f}(u ; \varepsilon)
$$

Corollary 2 (Gronwall estimates with Gevrey initial data). Let the assumptions of Theorem 1 hold, and additionally assume that (2.4) has only globally bounded solutions. Fix $R>0$, the maximal amplitude of the solution. Then for any $t_{0}>0$ there are constants $\varepsilon_{0}\left(t_{0}\right)>0$, and $C^{\prime}\left(t_{0}\right), c^{\prime}\left(t_{0}\right)>0$ such that the following holds.

Let $u(t)$ be a solution to the truncated equation (4.30) with norm bounded by $R$ in the Gevrey space $\mathcal{G}_{t^{*}, 1 / 2}$, for a time interval $0 \leq t \leq t_{0}<\infty$.

Then there exists a unique solution $v(t)$ on $0 \leq t \leq t_{0}$ to (4.28) with initial value $u(0)$. Moreover, the solutions are exponentially close in $\varepsilon<\varepsilon_{0}$,

$$
|v(t)-u(t)|_{X} \leq C^{\prime} \exp \left(-c^{\prime} \varepsilon^{-1 / 3}\right)
$$

for all $0 \leq t \leq t_{0}$. 
Proof. The difference $w(t)=v(t)-u(t)$ satisfies the equation

$$
\partial_{t} w=A(t) w+r(t)
$$

where

$$
A(t)=A+\int_{0}^{1}\left(\partial_{u} f+\partial_{u} \bar{f}\right)(\tau u+(1-\tau) v) \mathrm{d} \tau
$$

and

$$
r(t)=r(v(t))-r(u(t)),
$$

where for the sake of notation, we omitted the arguments $t$ and $\varepsilon$. Since $f, \bar{f}$, and $r$ possess bounded derivatives on bounded sets of $\mathcal{G}_{t^{*}, 1 / 2}$, the result is an immediate consequence of a standard Gronwall lemma. Note that Gevrey initial data will stay in the Gevrey space $\mathcal{G}_{t^{*}, 1 / 2}$, such that there is no transient in the exponential estimate. We also note that the estimate on the remainder $r$ is in the $X$-topology, only, such that the closeness result only holds in this topology. Furthermore when starting with arbitrary initial data, the transient in the exponential estimate would destroy an exponential estimate.

In the next theorem, we use smooth initial data in $\mathcal{G}_{\sigma, 1 / 2}$, which is defined in the same way as in (4.24) with the same function $\Gamma_{\sigma, \nu}$ as in (4.29). We denote a ball of radius $R$ in a Banach space $Y$ by $B_{R}(Y)$.

Theorem 3 (Exponential averaging of Gevrey regular solutions of nonlinear Schrödinger equations [MS03]). Consider equation (2.5) with analytic nonlinearities $C, g$. Assume $\Omega(u) \geq c_{0}>0$ for all $u \in X, \phi \in T^{1}$. Consider initial data in $\mathcal{G}_{\sigma, 1 / 2}$. There exists a near-identity transformation $I+\varepsilon W$, defined on the ball $B_{R}(X) \times T^{1}$, which eliminates adiabatically the fast phase, up to an exponentially small non-adiabatic effect and which is analytic on $B_{R}\left(\mathcal{G}_{\sigma, 1 / 2}\right) \times T^{1}$. In the new variables $(v, \psi)$, the evolution equation reads

$$
\begin{aligned}
i \partial_{t} v & =\Delta v+\bar{C}(v ; \varepsilon)+r^{1}(v, \psi ; \varepsilon), \\
\partial_{t} \psi & =\frac{1}{\varepsilon}(\Omega(v)+\tilde{\Omega}(v ; \varepsilon))+r^{2}(v, \psi ; \varepsilon) .
\end{aligned}
$$

The transformed nonlinearities $r^{1}, r^{2}, \bar{C}$ and $\tilde{\Omega}$ are bounded on the ball $B_{R}(X)$ and $B_{R}\left(\mathcal{G}_{\sigma, 1 / 2}\right)$ respectively, uniformly in $0<\varepsilon<\varepsilon_{0}$.

The non-adiabatic interaction terms $r^{1}$ and $r^{2}$ are exponentially small in $\varepsilon$, i.e., there exist constants $c, C>0$ such that

$$
\left|r^{1}(v, \psi ; \varepsilon)\right|_{X}+\left|r^{2}(v, \psi ; \varepsilon)\right|<C \exp \left(-c \varepsilon^{-1 / 3}\right),
$$

for all $v \in B_{R}\left(\mathcal{G}_{\sigma, 1 / 2}\right)$, and all $\psi \in T^{1}$. The adiabatic corrections $\bar{C}$ and $\tilde{\Omega}$ are small in Gevrey spaces,

$$
|\tilde{C}(v ; \varepsilon)|_{\mathcal{G}_{\sigma, 1 / 2}} \leq C \varepsilon^{1 / 3}, \quad|\tilde{\Omega}(v ; \varepsilon)| \leq C \varepsilon
$$

for $v \in B_{R}\left(\mathcal{G}_{\sigma, 1 / 2}\right)$. 
Proof. A detailed proof for general evolution equation or Hamiltonian partial differential equations can be found in [MS03]. The proof is similar to the proof of theorem 1. The formal transformations we consider are given in (3.19). Estimates on the remainder term in approximation space can be derived in the same way as above. For the analysis of the error due to the Galerkin space, we use that the initial data are in $\mathcal{G}_{\sigma, 1 / 2}$ and that $(2.5)$ is well-posed on this space for finite times. Then the estimates on the non-adiabatic remainder in $P_{N} B_{R}$ and the error of the Galerkin approximation can be balanced again to obtain the desired exponential estimate.

A comparable corollary of Gronwall type also holds.

Theorem 4 (Averaging of Gevrey regular solutions of parabolic equations under quasiperiodic forcing [Mat04]). Consider the reaction-diffusion equation (2.4) with analytic nonlinearities and $\Omega(u, \phi)=\omega$ with Diophantine conditions (3.20). Then, for any ball of radius $R$ in $\mathcal{G}_{\sigma, \nu}$ there exists an $\varepsilon_{0}>0$, such that for $0<\varepsilon<\varepsilon_{0}$ there exists a near identity transformation of both $\mathcal{G}_{\sigma, 1 / 2}$ and $X$ to

$$
\begin{aligned}
\partial_{t} v & =\Delta v+f(v)+\bar{g}(v, \varepsilon)+r(v, \psi, \varepsilon) \\
\partial_{t} \psi & =\frac{1}{\varepsilon} \omega
\end{aligned}
$$

with initial conditions $v(0)=u_{0} ; \theta(0)=\theta_{0}$ and with $\bar{g}$ and $r$ both bounded on balls in $X$, furthermore the remainder term is exponentially small on balls of the Gevrey space.

$$
\begin{aligned}
|\bar{g}(v, \varepsilon)|_{X} & \leq C \varepsilon^{(\tau+1) /(\tau+3)} \\
|r(v, \theta, \varepsilon)|_{X} & \leq C\left(|v|_{\mathcal{G}_{\sigma, \nu}}\right) \exp \left(-c \varepsilon^{-1 /(\tau+3)}\right) .
\end{aligned}
$$

Details, variants and extensions are given in [Mat04].

Proof. A major part is already the estimate on the approximation space, see [Sim94]. The remainder on the approximation space is of order $\mathcal{O}\left(\exp \left(-c \varepsilon^{(\varepsilon N(\varepsilon))^{1 /(1+\tau)}}\right)\right)$. The error estimate of the Galerkin approximation is as above of order $\mathcal{O}\left(\exp \left(-c N(\varepsilon)^{1 / 2}\right)\right.$, such that the optimal coupling is $N(\varepsilon)=$ $\varepsilon^{-2 /(3+\tau)}$, which yields the exponential estimate.

Theorem 5 (Homogenisation via spatial dynamics [Mat05]). Consider (2.7) with analytic nonlinearity $f(.,$.$) , which is periodic in the second component. Then there exist \varepsilon_{0}>0, c, C>0$ and a t-periodic transformation of (2.8) on a ball $B_{R}(X)$ for $0<\varepsilon<\varepsilon_{0}$ to

$$
V_{t}=A V+F(V)+\bar{F}(V, \varepsilon)+r(V, t / \varepsilon, \varepsilon),
$$

where $\bar{F}, \alpha$ are differentiable for $V \in B_{R}(X)$, nonlinear and nonlocal in the cross-section, but local in $t$. When considering bounded solutions $V(.) \in B C(\mathbf{R}, X)$ of the original equation (2.8) then the influence of the fast scale on $V($.$) is exponentially small, uniformly on balls in B C(\mathbf{R}, X)$ :

$$
\|r(V(.), . / \varepsilon, \varepsilon)\|_{B C(\mathbf{R}, X)} \leq C \exp \left(-c \varepsilon^{-1 / 2}\right) .
$$


The correction term can be estimated on $B_{R}(X)$ by

$$
\sup _{V(.) \in B_{X}(R)}\|\bar{F}(V(.), \varepsilon)\|_{B C(\mathbf{R}, X)} \leq C \varepsilon .
$$

Proof. A complete proof of this homogenisation and of variants can be found in [Mat05, MW06]. The estimates on the approximation space are similar to the estimates in theorem 1 . Using again the coupling

$$
N(\varepsilon) \varepsilon^{\alpha}=1 .
$$

Then we use again a regularity result. We let $A$ as in (2.8) and use

$$
\Gamma_{\sigma, \nu}=\exp \left(-\sigma|A|^{\nu}\right)
$$

in the definition of the Gevrey norm in (4.24). Then we obtain that the globally bounded solutions are in fact highly regular as functions on the cross-section $\Omega$

$$
V(t) \in \mathcal{G}_{\sigma_{*}, 1}
$$

for some $\sigma_{*}>0$ with estimates uniform in $t$. The set of all such solutions is sometimes called the attractor of the spatial dynamics equation. Then the overall remainder on bounded sets within the attractor can be bounded by

$$
\begin{aligned}
& \left|r_{k(\varepsilon)}\right| X+\left|G\left(V(t)-P_{N(\varepsilon)} V(t)\right)\right|_{X} \\
\leq & C \exp \left(-c \varepsilon^{-1+\alpha}\right)+C_{0} \exp \left(-\sigma_{*} / N(\varepsilon)\right) \\
= & C \exp \left(-c \varepsilon^{-1+\alpha}\right)+C_{0} \exp \left(-\sigma_{*} \varepsilon^{-\alpha}\right) .
\end{aligned}
$$

Choosing $\alpha=1 / 2$ yields the result for appropriate $C$ and $c$. The results hold in the same way for any function $V($.$) , which is smooth enough, e.g. by being a solution of a similar averaged$ equation.

Theorem 6 (Homogenisation of elliptic operators [KMS06]). Suppose $\mathcal{A} \in L^{\infty}\left(T^{d}\right), f \in \mathcal{G}_{\sigma, 1 / 2}$ (as defined above) and $\int_{T^{d}} f=0$ in equation (2.9). Let $u^{\varepsilon}$ be the solution. Then there exist $\varepsilon$ independent constants $C>0, c>0, \kappa>0$, such that for any $N \sim \kappa \varepsilon^{-1}$ the approximation (3.22) has the error bound:

$$
\left\|u^{\varepsilon, N}-u^{\varepsilon} ; H^{1}(\mathbb{T})\right\| \leq C \exp \left(-c \varepsilon^{-1}\right)
$$

The proof in [KMS06] is based on a careful analysis of the remainder term $u^{\varepsilon, k}-u^{\varepsilon}$, then the error can be estimated in an exponential way for $k=N \sim \kappa \varepsilon^{-1}$, where only regularity of $f$ is needed, but not on the regularity of the matrix $\mathcal{A}$.

\section{EFFECts}

In this section we discuss some effects due to the heterogeneous or nonautonomous structure of the equation. 
5.1. Splitting of homoclinic orbits. An important difference in the dynamics between autonomous and non-autonomous differential equation are the existence of transversal homoclinic orbits. So consider a parameter dependent version of (2.4) without rapid forcing

$$
\partial_{t} u=\Delta u+f(u, \lambda)
$$

with $\lambda \in \mathbf{R}$. A homoclinic orbit is a solution, which is biasymptotic for $t \rightarrow \pm \infty$ to some hyperbolic equilibrium $u_{0}$, i.e. $\Delta u_{0}+f\left(u_{0}, \lambda\right)=0$ and the operator $\Delta+D f\left(u_{0}, \lambda\right)$ has only spectrum away from the imaginary axis. Under some non-degeneracy conditions, this homoclinic orbit will only exist for special values $\lambda_{0}$. Whereas in the externally forced equation

$$
\partial_{t} u=\Delta u+f(u, \lambda)+g(u, t / \varepsilon)
$$

the stable and unstable manifold will intersect transversally, creating rich dynamics nearby. The main assertion of theorem 1 is, that the nonautonomous dynamics of (5.36) can be described by the exponentially close autonomous equation as in (4.30). It is possible to show by some further analysis that the equilibrium persists as a hyperbolic periodic orbit, exponentially close to an equilibrium of the truncated equation after the transformation of theorem 1. Then also the phase-portraits with unstable and parts of the stable manifolds will be exponentially close, see [Mat01]. From this, it is easy to see, that transversality effects can only occur in a small parameter interval $\left(\lambda^{-}(\varepsilon), \lambda^{+}(\varepsilon)\right)$ with

$$
\left|\lambda^{+}(\varepsilon)-\lambda^{-}(\varepsilon)\right| \leq C \exp \left(-c \varepsilon^{-1 / 3}\right)
$$

such that these effects were called "invisible chaos" [FS96].

Using functional analytic methods as in [Mat03] one obtains better results for large classes of parabolic equations, by analysing the problem in complex time. A more detailed analysis is possible for ordinary differential equations including lower estimates, see e.g. [Gel99, HMS88].

5.2. Pinning. Pinning describes a phenomenon in equations like (2.6), where a travelling wave does not propagate due to heterogeneous structures in the medium. Thus travelling waves will be instead standing waves, i.e. they are solutions to (2.7). A pinned wave is then homoclinic or heteroclinic orbit in the spatial dynamics setting (2.8). Pinning occurs when there a transversal intersection of stable and unstable manifolds. The general idea is similar to the analysis of the transversal intersection above. Here it is more convenient to find solutions nearby a pulse $U_{0}$ as zeros of

$$
I(V)=\partial_{t}\left(U_{0}+V\right)-A\left(U_{0}+V\right)-F\left(U_{0}+V\right) \in B C(\mathbf{R}, X)
$$

Using Fredholm properties or exponential dichotomies, the problem is reduced to a low-dimensional problem, where the effects of the heterogeneous terms can be estimated to be of order $\mathcal{O}\left(\exp \left(-c \varepsilon^{-1 / 2}\right)\right)$, see $[\mathrm{MW06}]$. 


\section{Discussion}

In this article we have given an overview on results about averaging and homogenisation for partial differential equations beyond every order. The first step of this analysis are finite-order averaging estimates, which were iterated, to obtain exponential estimates on the transformed equation. With Gronwall-type estimates, we can extend this to estimates about the finite time behaviour. For particular solutions, equilibria, stable and unstable manifolds and connecting orbits the analysis can be extended to infinite times too. Due to the general nature of the procedure there remain several problem, which require a finer analysis.

The construction of the transformation and the remainder terms are iterative so they are not easily computable, but they coincide to finite order with their finite order counter-parts. Therefore, it is possible to show by direct calculations, that the transformed nonlinearities are typically not-local in $x$, even if the original nonlinearities are local, see [Mat04].

A very subtle point are lower estimates for both averaging procedures and particular effects. For the general averaging procedure, there is an example in [MS03], showing that the averaging results cannot be improved by any other "averaging-type" transformation. In particular there is difference between ordinary differential equations and infinite dimensional systems in what kind of exponents can be achieved by exponential averaging. In [KMS06], a particular simple choice of the heterogeneous matrix allows explicit calculations and shows, that the truncation of the asymptotic expansion is optimal.

For particular effects like the splitting of homoclinic orbits, there are several ways to obtain lower estimates in the finite dimensional case, see [HMS88, Gel99]. A crucial part is extending the analysis to complex time and analysing the time-singularities in the complex plane. This idea is also used in

other cases of exponential analysis [BT05, CM05] and references therein. Some ways of introducing exponential asymptotics into numerical analysis can be found in [MBS00, Mat03]. The relation of exponential averaging and integrable systems is still open, but see [Bam99, Poe99] for related results.

The analysis here was restricted to cases to some partial differential equations with periodic boundary conditions, the needed abstract properties, such that the theorems hold for large classes of evolution equations are given e.g. in [MS03, Mat04]. Then these results will hold e.g. for problems on the domain $\mathbf{R}^{d}$. The effect of boundary conditions and of boundary layers [Neu00] on exponential homogenisation still remains to be analysed.

Acknowledgements. This work has been supported by the DFG Priority Program 1095 "Analysis, Modelling and Simulation of Multiscale Problems" under Fi 441/12. The author is grateful to Bernold Fiedler for support and encouragement during the work on this project at Freie Universiät Berlin. 


\section{REFERENCES}

[AM95] A. Afendikov, and A. Mielke. Bifurcations of Poiseuille flow between parallel plates: three-dimensional solutions with large spanwise wavelength. Arch. Rational Mech. Anal., 129(2):101-127, 1995.

[AKN97] V. Arnold, V. Kozlov, and A. Neishtadt. Mathematical Aspects of Classical and Celestial Mechanics. Springer Verlag, Berlin, 1997.

[BP84] N.S. Bakhvalov, and G.P. Panasenko. Homogenization: Averaging Processes in Periodic Media. Nauka, Moscow 1984. (in Russian). English translation in: Mathematics and Its Applications (Soviet Series) 36, Kluwer Academic Publishers, Dordrecht-Boston-London 1989.

[Bam99] D. Bambusi. Nekhoroshev theorem for small amplitude solutions in nonlinear Schrödinger equations. Math. Z., 230:345-387, 1999.

[Bam03a] D. Bambusi. An averaging theorem for quasilinear Hamiltonian PDEs. Ann. Henri Poincar 4(4):685$712,2003$.

[Bam03b] D. Bambusi. Birkhoff normal form for some nonlinear PDEs. Comm. Math. Phys. 234(2):253-285, 2003.

[Bam06] D. Bambusi. Galerkin averaging method and Poincaré normal form for some quasilinear PDEs. Ann. Scuola. Norm. Sup. Pisa to appear, 2006.

[BLP78] A. Bensoussan, J.-L. Lions, and G.-C. Papanicolaou. Asymptotic analysis for periodic structures. North Holland, Amsterdam, 1978.

[BT05] V. Betz, and S. Teufel. Precise coupling terms in adiabatic quantum evolution: the generic case Comm. Math. Phys., 260:481-509, 2005.

[BHR05] H. Berestycki, F. Hamel, and L. Roques. Analysis of the periodically fragmented environment model. II. Biological invasions and pulsating travelling fronts. J. Math. Pures Appl. (9), 84:1101-1146, 2005.

[BoMi61] N. Bogoliubov, and Y. Mitroploski. Asymptotic Methods in the theory of nonlinear oscillations, Gordon and Breach, New York, 1961.

[Bor98] F. Bornemann. Homogenization in time of singularly perturbed mechanical systems. Lecture Notes in Mathematics 1687, Springer-Verlag, Berlin, 1998.

[CS04] K.D. Cherednichenko, and V.P. Smyshlyaev. On full two-scale expansion of the solutions of nonlinear periodic rapidly oscillating problems and higher-order homogenized variational problems. Arch. Ration. Mech. Anal., 174:385-442, 2004.

[CM05] S.J. Chapman, and D.B. Mortimer. Exponential asymptotics and Stokes lines in a partial differential equation. Proc. R. Soc. Lond. Ser. A Math. Phys. Eng. Sci. 461:2385-2421, 2005.

[FT98] A.B. Ferrari, and E.S. Titi. Gevrey regularity for nonlinear analytic parabolic equations. Comm. Part. Diff. Eq., 23(1-2):1-16, 1998.

[FS03] B. Fiedler, and A. Scheel. Spatio-temporal dynamics of reaction-diffusion patterns. in Kirkilionis, Markus (ed.) et al., Trends in nonlinear analysis. On the occasion of the 60th birthday of Willi Jger. Berlin: Springer. 23-152, 411-417, 2003.

[FS96] B. Fiedler, and J. Scheurle. Discretization of homoclinic orbits, rapid forcing and "invisible" chaos. Mem. Amer. Math. Soc., 119, 1996.

[FV01] B. Fiedler, and M. Vishik. Quantitative homogenization of analytic semigroups and reaction diffusion equations with diophantine spatial frequencies. Adv. Differ. Equ., 6:1377-1408, 2001.

[Gel99] V. Gelfreich. A Proof of the Exponentially Small Transversality of the Separatrices for the Standard Map. Comm. Math. Phys., 201: 155-216, 2000.

[GMMP97] P. Gérard, P. Markowich, N.J. Mauser, and F. Poupaud. Homogenization limits and Wigner transforms. Comm. Pure Appl. Math., 50: 323-379, 1997. 
[HMS88] P. Holmes, J. Marsden and J. Scheurle. Exponentially small splittings of separatrices with applications to KAM theory and degenerate bifurcations. Hamiltonian dynamical systems (Boulder, CO, 1987), Contemp. Math., 81: 213-244, Amer. Math. Soc., Providence, RI, 1988.

[IM91] G. Iooss, and A. Mielke. Bifurcating time-periodic solutions of Navier-Stokes equations in infinite cylinders. J. Nonlinear Science, 1:107-146, 1991.

[JKO94] V.V. Jikov, S.M. Kozlov, and O.A. Oleinik. Homogenization of Differential Operators and Integral Functionals. Springer-Verlag, Berlin, 1994.

[KMS06] V. Kamotski, K. Matthies, and V. Smyshlyaev. Exponential homogenization of linear second order elliptic problems with periodic coefficients BICS Preprint 2/2006.

[Kee00] J.P. Keener. Propagation of waves in an excitable medium with discrete release sites. SIAM J. Appl. Math., 61(1):317-334, 2000.

[KS98] J.P. Keener, and J. Sneyd. Mathematical physiology. Springer, New York, 1998.

[Kir82] K. Kirchgässner. Wave solutions of reversible systems and applications. J. Diff. Eq., 45:113-127, 1982.

[Kr89] M.S. Krol. On a Galerkin-averaging method for weakly nonlinear wave equations. Math. Methods Appl. Sci., 11:649-664, 1989.

[LM88] P. Lochak, and C. Meunier. Multiphase Averaging for Classical Systems, Appl. Math. Sc. 72, SpringerVerlag, New York 1988.

[MBS00] A.M. Matache, I. Babuška, and C. Schwab. Generalized p-FEM in homogenization. Numer. Math. $86: 319-375,2000$.

[Mat01] K. Matthies. Time-averaging under fast periodic forcing of parabolic partial differential equations: exponential estimates. J. Differential Equations, 174(1):133-180, 2001.

[Mat03] K. Matthies. Exponentially small splitting of homoclinic orbits of parabolic differential equations under periodic forcing. Discrete Contin. Dyn. Syst., 9(3):585-602, 2003.

[Mat03] K. Matthies. Backward error analysis of a full discretisation scheme for a class ofparabolic partial differential equations Nonlinear Anal., 52(3):805-826, 2003.

[MS03] K. Matthies, and A. Scheel. Exponential Averaging of Hamiltonian Evolution Equations, Trans. Amer. Math. Soc., 355, 747-773, 2003.

[Mat04] K. Matthies. Exponential Averaging of Rapid Quasiperiodic Forcing, preprint, 2004.

[Mat05] K. Matthies. Homogenization of exponential order for elliptic systems in infinite cylinders. Asymptot. Anal. 43:205-232, 2005.

[MW06] K. Matthies, and C.E. Wayne. Wave pinning in strips. Proc.Roy. Soc. Edinburgh A, to appear 2006.

[MSU06] K. Matthies, G. Schneider, and H. Uecker. Exponential averaging and traveling waves in rapidly varying periodic media Mathematische Nachrichten, to appear 2006.

[Nei84] A.I. Neishtadt. The separation of motions in systems with rapidly rotating phase. J. Appl. Math. Mech., 48:133-139, 1984.

[Nek79] N. N. Nekhorošev, An exponential estimate of the time of stability of nearly integrable Hamiltonian systems, (Russian) Uspehi Mat. Nauk, 32:5-66, 1977 .

[Neu00] A. Neuss-Radu. A result on the decay of the boundary layers in the homogenization theory. Asymptot. Anal. 23:313-328, 2000.

[NM92] A. Newell, J. Moloney. Nonlinear Optics, Advanced Topics in the Interdisciplinary Sciences, AddisonWesley, Redwood City, 1992.

[Poe99] J. Pöschel. On Nekhoroshev estimates for a nonlinear Schrödinger equation and a theorem by Bambusi, Nonlinearity 12:1587-1600, 1999. 
[Pro91] K. Promislow. Time analyticity and Gevrey regularity for solutions of a class of dissipative partial differential equations. Nonlinear Anal., 16(11):959-980, 1991.

[RMMSC03] S. Rüdiger, D.G. Miguez, A.P. Munuzuri, F. Saguesa, and J. Casademunt. Dynamics of Turing patterns under spatio-temporal forcing. Phys. Rev. Lett., 90(12):128301, 2003.

[SV85] J.A. Sanders, and F. Verhulst. Averaging methods in nonlinear dynamical systems. Applied Mathematical Sciences 59, Springer, New York, 1985.

[SaScWu99] B. Sandstede, A. Scheel, and C. Wulff. Bifurcations and dynamics of spiral waves. J. Nonlinear Science, 9:439-478, 1999.

[Sim94] C. Simó. Averaging under fast quasiperiodic forcing, in Hamiltonian Mechanics (Torun, 1993), NATO Adv. Sci. Inst.Ser. B Phys. 331:13-34, 1994.

[TBDHT96] P. Takáč, P. Bollerman, A. Doelman, A. van Harten, and E. S. Titi. Analyticity of essentially bounded solutions to semilinear parabolic systems and validity of the Ginzburg-Landau equation. SIAM J. Math. Anal., 27(2):424-448, 1996.

[Tar79] L. Tartar. Compensated compactness and applications to partial differential equations. in: Nonlinear analysis and mechanics: Heriot-Watt Symp., Vol. 4, Edinburgh 1979, Res. Notes Math., 39: 136-212, 1979.

[Tar86] L. Tartar. Oscillations in nonlinear partial differential equations: compensated compactness and homogenization, in: Nolinear systems of partial differential equations in applied mathematics, Lectures in Applied Mathematics 74, AMS, Providence, 243-266, 1986.

[Van89] A. Vanderbauwhede. Centre manifolds, normal forms and elementary bifurcations. in:Dynamics reported, Vol. 2, Wiley, Chichester, 89-169, 1989.

[Ver05] F. Verhulst. Methods and applications of singular perturbations. Boundary layers and multiple timescale dynamics. Texts in Applied Mathematics, 50. Springer, New York, 2005.

[Ver06] F. Verhulst. On averaging methods for partial differential equations preprint, 2006.

[Xin00] J. Xin. Front propagation in heterogeneous media. SIAM Rev., 42(2):161-230, 2000.

University of Bath, Department of Mathematical Sciences, Bath, BA2 7AY, United Kingdom

E-mail address: K.Matthies@maths.bath.ac.uk 